\title{
Mediterranean plant karyological data - 31
}

\author{
edited by G. Kamari, C. Blanché \& S. Siljak-Yakovlev
}

\begin{abstract}
Kamari, G., Blanché, C. \& Siljak-Yakovlev, S. (eds): Mediterranean plant karyological data 31. — F1. Medit. 31: 335-354. 2021. — ISSN: 1120-4052 printed, 2240-4538 online.

This is the thirty-one of a series of karyological data from Mediterranean area, peri-Alpine communities and the Atlantic Islands, in English or French language. It comprises contributions on 11 taxa: Hieracium from Sicily and S Italy by E. Di Gristina, G. Domina, \& A. Geraci (Nos 1997-1999); Allium, Drimia, Oncostema and Pancratium from Tunisia by G. Barone, E. Di Gristina, R. El. Mokni \& G. Domina (Nos 2000-2003); Allium, Cyclamen, Cynara and Ornithogalum from Greece, Crete and East Aegean Islands by E. Liveri, D. Phitos \& G. Kamari (Nos 2004-2007).
\end{abstract}

Addresses of the editors:

Prof. Georgia Kamari, Botanical Institute, Section of Plant Biology, Department of Biology, University of Patras, GR-26500 Patras, Greece. E-mail: kamari@upatras.gr

Prof. Cesar Blanché, IRBio-GReB, Laboratori de Botànica, Facultat de Farmàcia, Universitat de Barcelona, Av. Joan XXIII s/n, E-08028 Barcelona, Catalonia, Spain. E-mail: cesarblanche@ub.edu

Dr. Sonja Siljak-Yakovlev, Université Paris-Saclay, CNRS, AgroParisTech, Ecologie Systématique Evolution, 91405, Orsay, France. E-mail: sonia.yakovlev@universite-paris-saclay.fr 